\title{
SENSOR FUSION ALGORITHMS FOR THE DETECTION OF LAND MINES
}

\author{
M.G.J. Breuers, P.B.W. Schwering, S.P. van den Broek \\ TNO Physics and Electronics Laboratory, P.O. Box 96864, \\ 2509 JG The Hague, The Netherlands
}

\begin{abstract}
This paper reports on selection of suitable sensor fusion techniques for use in hand carried and vehicle mounted mine detection systems for HOM-2000. Various techniques like Bayes, Dempster-Shafer, voting logic and neural networks are considered. Guidelines for selection of an appropriate fusion level are formulated. Experimental data are presented to illustrate the advantages that can be obtained through the use of sensor fusion.
\end{abstract}

Keywords: land mine detection, sensor fusion, HOM-2000

\section{INTRODUCTION}

The use of multi-sensor technology has become part of a growing number of national and international research programs that seek to develop more efficient and reliable mine detection techniques. The HOM-2000 project is a Dutch government initiative focusing on the development of both hand carried and vehicle mounted mine detection systems that achieve improved performance through simultaneous use of several different sensor types. Various sensor combinations and sensor fusion options have been examined during phase A of the HOM-2000 project.

Part of HOM-2000 phase A is a feasibility study on sensor fusion techniques. Various aspects of sensor fusion are taken into account in this project: feature extraction from raw sensor signals, co-registration of sensor signals and sensor management. In this paper we will first discuss some of the candidate fusion techniques that have been examined. Finally, we will present images that were gathered by GPR (ground penetrating radar), EMI (electromagnetic induction) and TIR (thermal infrared) sensors at the TNO-FEL test facility to illustrate the benefits that can be obtained through sensor fusion.

\section{FUSION MECHANISMS}

Sensor fusion is considered to be a method that can help to combine the strengths of individual sensors and to create a sensing system that as a whole achieves better performance than any of the individual sensors. Among the expected advantages are improved detection, improved classification, wider sensing range, increased robustness, and increased spatial resolution. In this section we will discuss these advantages and the mechanisms behind them. Understanding how these mechanisms apply to the sensor configuration will help in selecting a suitable fusion technique.

In the process of sensor fusion, improvement of the detection and classification performance can be obtained through

different mechanisms. The three most important mechanism for our application are:

1) The combined depth range that is covered by a set of sensors can be larger than the depth range that is covered by any of the individual sensors.

2) The mean value of 'compatible' sensor readings will have on average a better signal to noise ratio than individual sensors in situations where the individual sensors are corrupted by uncorrelated disturbances.

3) Classification can be improved if several different features of an object that together describe a unique object class can be sensed simultaneously.

In the mine detection scenarios under study we expect that sensor fusion advantages are mainly achieved through mechanisms 1 and 2. This follows from the sensor characteristics that are shown in table 1. TIR and GPR for example, will in general observe different depth ranges. This means they are complementary sensors that can aid in fusion through mechanism 1.

The. performance of all sensors is to some extend affected by the moisture content of the soil. A moderate moisture level can decrease the performance of the GPR sensor severely while the impact on EMI performance is only small. Increased soil 
moisture content can either enhance or diminish the detection performance of the TIR camera. Small amounts of moisture that easily evaporate on the surface under good weather conditions can help to increase the temperature contrast of the soil. However, high soil moisture content will decrease the temperature contrast of the soil. The differences in the way moisture affects the performance of the three sensors, indicate that performance gain can be achieved through sensor fusion mechanism 2.

Based on the performance of the sensors and feature extractors that are currently being evaluated in the HOM-2000 project, we expect that the number of features and the quality of the features that can be extracted is insufficient to enable reliable object classification through fusion mechanism 3. If a more extensive training set of sensor data becomes available, the feasibility of object classification can be reconsidered.

Table 1: Characteristics properties of EMI, GPR and TIR sensors

\begin{tabular}{|r|c|c|c|}
\hline & EMI & GPR & TIR \\
\hline Observed depth range & $\begin{array}{c}\text { above surface } \\
\text { surface interface } \\
\text { below interface }\end{array}$ & above surface & $\begin{array}{c}\text { above surface } \\
\text { surface interface }\end{array}$ \\
\hline Observed quantity & electrical conductivity & dielectric constant & $\begin{array}{c}\text { thermal capacity } \\
\text { thermal emissivity }\end{array}$ \\
\hline Influence of soil moisture & decreased performance & strongly decreased performance & $\begin{array}{c}\text { decreased performance } \\
\text { or }\end{array}$ \\
Extracted features & $\begin{array}{c}\text { object material } \\
\text { (object size) }\end{array}$ & $\begin{array}{c}\text { object material } \\
\text { object size }\end{array}$ & $\begin{array}{c}\text { object size } \\
\text { object shape } \\
\text { object surface structure }\end{array}$ \\
\hline
\end{tabular}

\section{FUSION LEVELS}

The choice of a suitable fusion level is important since it affects both the quality of the detection results and complexity of the fusion process. Three different fusion levels' can be discerned: data level, feature level and decision level. Information at data level consists of pixel values in camera images and samples of raw waveforms early in the detector signal processing chain. Information at feature level consists of object properties like size and material type. Information at decision level consists of numbers that represent the probability of presence of a certain object type.

Fusion at data level is often applied to groups of similar sensors, such as cameras that are sensitive in different wavelength ranges. Fusion at feature level is most suitable in object classification applications. Fusion at decision level can easily be applied to very different sensors systems.

The choice of an appropriate fusion level is guided by the following observations. On the one hand, fusion at low level can in some cases prevent information loss that might occur when moving up to higher fusion levels. On the other hand, choosing a higher fusion level reduces the computational complexity of the fusion process and also eases the tasks of fusing information from very different sensor types. Therefore, sensor fusion should take place at the highest level at which the amount of information loss is still acceptable.

Fusion mechanism 1 is important in the HOM-2000 project. In principle, it can take place at any level without loss of information, so fusion should be done at the highest level, that is, the decision level. Fusion mechanism 2 applies both at feature and at decision level, but moving to a higher level can mean information loss. However, in our case no suitable features are identified for this fusion mechanism, so this leaves the option of fusion at decision level. Fusion mechanism 3 only applies at feature level, but since no suitable features are available to support this type of fusion we can rule out this type of fusion. Based on these arguments we choose to focus on fusion at decision level in phase A of HOM-2000.

\section{FUSION ALGORITHM}

Fusion algorithms can be divided into two classes, based on whether the decision process relies mainly on expert knowledge and understanding of the physics that governs the detection processes, or on statistical modelling of experiment data. In the 
sensor fusion project we have taken into consideration the fusion techniques that are shown in table 2 . We will discus some advantages and disadvantages that are related to these techniques.

The Bayesian approach guarantees, within the limitations that are dictated by the selected set of sensors and feature extraction algorithms, an optimal estimate of the environment that is visible to the sensors, if a perfectly reliable model of sensor and environment is available.

Table 2: Sensor-fusion techniques.

\begin{tabular}{|l|l|}
\hline STATISTICAL / & EXPERT KNOWLEDGE \\
BLACK-BOX MODEL & \\
\hline - Bayesian & - Fuzzy logic \\
- Dempster-Shafer & - Expert system \\
- Neural network & \\
- Weighted voting procedure & \\
\hline
\end{tabular}

The Dempster-Shafer technique is very similar to the Bayesian approach, but it is more effective if information is available about the relative accurateness of various parts of the statistical model that is used to describe the sensors. It can be shown that under certain conditions the Dempster-Shafer approach and the Bayesian technique are identical. Also, the Dempster-Shafer technique is very flexible since sensors can be easily added without adjusting the probability masses of the remaining sensors.

Neural networks can be used to create arbitrary functional mappings. In order to achieve reliable performance a large number of training samples has to be presented to a neural network. The need for large training sets is a serious drawback of neural networks.

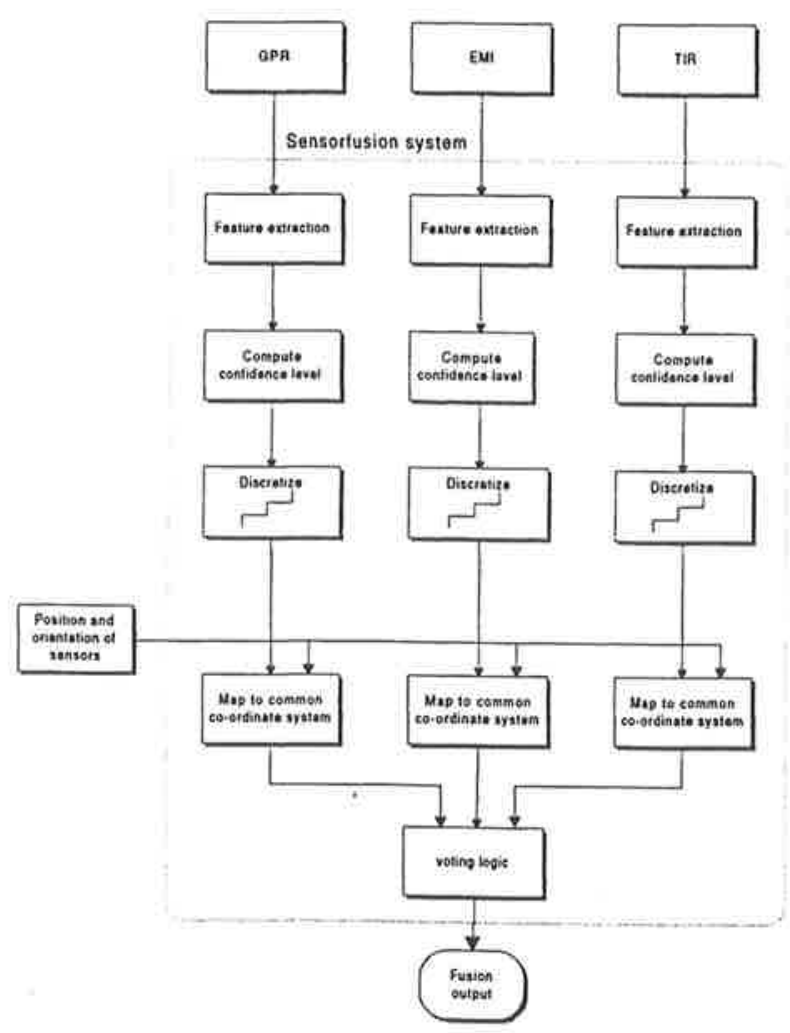

Figure I: Main processing steps in the mine detection system. 
Expert systems are tools that can be used to represent expert knowledge as a set of rules. The set of rules is applied to input data by means of an interference mechanism in order to verify certain hypothesis. In a de-mining scenario a hypothesis might for example state the existence of a mine at a certain location.

Fuzzy logic also relies on expert knowledge and a database of rules. A major difference between these two techniques is that fuzzy logic does not use a forward or backward chaining interference mechanism that links rules in sequential manner. Moreover, fuzzy logic is more suited to model continuous function mappings.

A weighted voting procedure ${ }^{6,7}$ incorporates the intuitive notion that a hypothesis becomes more likely if more supporting evidence is available. The voting rules can be used to express that weak support of a hypothesis from several sensors is equivalent to strong support from a single sensor. In addition, the relative reliability of sensors can be modelled by weight coefficients.

Taking into account the advantages and disadvantages of various options that were described above, we think that a weighted voting procedure is a suitable technique for our specific needs. This choice is motivated by the fact that it is the most simple technique that can support the two basic fusion mechanisms that are involved in decision level fusion: complementary sensing depth range and uncorrelated sensor disturbance. Both of these mechanisms lead to the notion that strong evidence for the presence of a mine obtained from a single sensor should be sufficient to trigger a positive output from a sensor-fusion system that is tuned to achieve a high detection rate. The main processing steps that occur in the sensorfusion system are shown in Figure 1.

\section{EXPERIMENTS}

Part of the HOM-2000 project is the construction of an outdoor test facility containing various soil types in which realistic surrogate mines and decoy objects are buried ${ }^{9,10}$. The facility consists of six test lanes of $3 \times 10 \mathrm{~m}$ that are filled respectively with sand, clay, peat, high iron content soil, forest soil, and rocky soil. The groundwater level in each of the test lanes can be adjusted automatically to a user defined level. A completely non-metal platform on which sensors are mounted can be moved across the test lanes. Both mines and potential false alarms like tin cans, bottles, rocks, and metal fragments are placed in the test lanes. The facility is used in the selection of suitable sensors and for the generation of test data sets that can be used in the training and evaluation of sensor fusion techniques.

\subsection{Thermal infrared sensors}

Four different TIR FPA cameras, that are either sensitive in the 3-5 $\mu \mathrm{m}$ wavelength range or in the 8-12 $\mu \mathrm{m}$ wavelength range, are taken into consideration in the camera selection process. The spatial resolution of all detector arrays is $256 \times 256$ pixels. In one camera model, microscanning is employed to enhance the spatial resolution. The noise equivalent temperature difference is smaller than $25 \mathrm{mK}$.

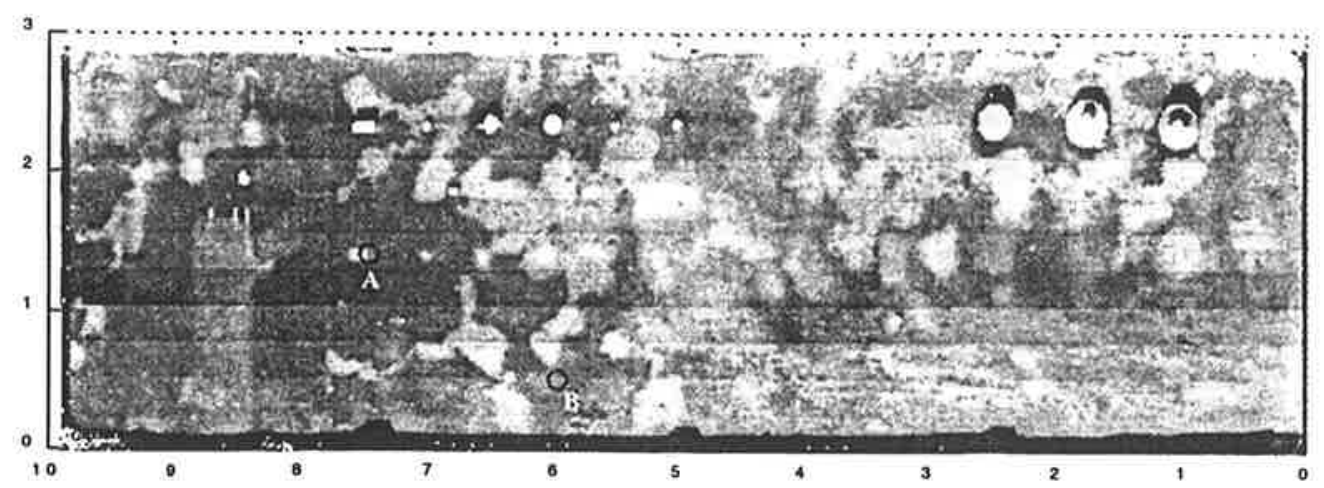

Figure 2. Mosaic of TIR images (8-12/m) from sandy soil test lane. Circles denote ground truth positions of mines. 
Automatic detection of mine-like targets is not an off-the-shelf technology for IR cameras. We have evaluated several options to achieve automatic object recognition, among which are template matching and texture extraction. After this initial processing step, size is used as an additional selection criterion to remove unlikely candidate image regions. Figure 2 shows a mosaic of TIR images that were collected from the sandy soil test lane.

\subsection{Ground penetrating radar}

Two hand carried and two vehicle carried radar systems are evaluated. All systems use pulsed signals. The vehicle carried systems consist of several send and receive antennas that enable beam forming and thus are likely to produce more detailed images.

The first step in the GPR based detection process consists of background removal using a moving average filter. To some extend the background removal is also helpful in suppression of reflections that occur at the air-ground interface. The cumulative energy of all reflections is projected towards the surface plane. A typical image that results from this processing step is shown in Figure 3. Finally, suspicious regions are selected based on local contrast and size.

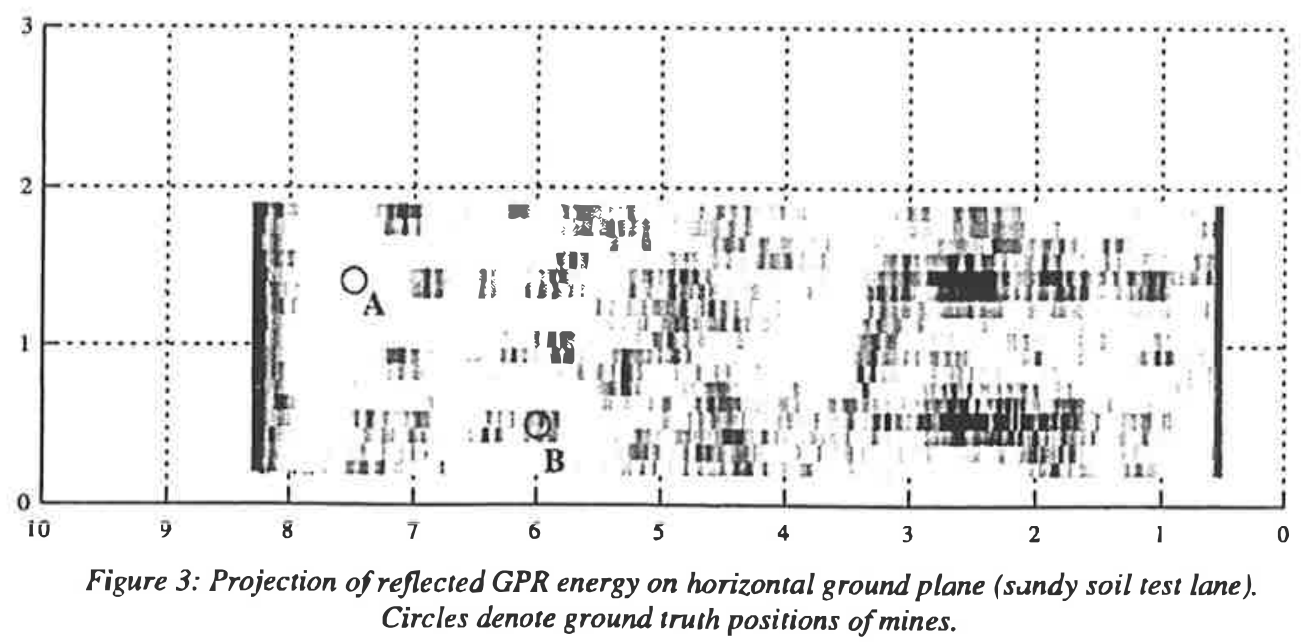

\subsection{Electromagnetic induction sensors}

Both pulse induction and continuous-wave sensors are tested. In the pulse detection sensors, the decay time of the received antenna signal is used as a discriminating feature. In the dual-frequency continuous-wave sensor the amplitude difference between two received sinusoidal signals of different frequency is used to discriminate metallic objects from background.

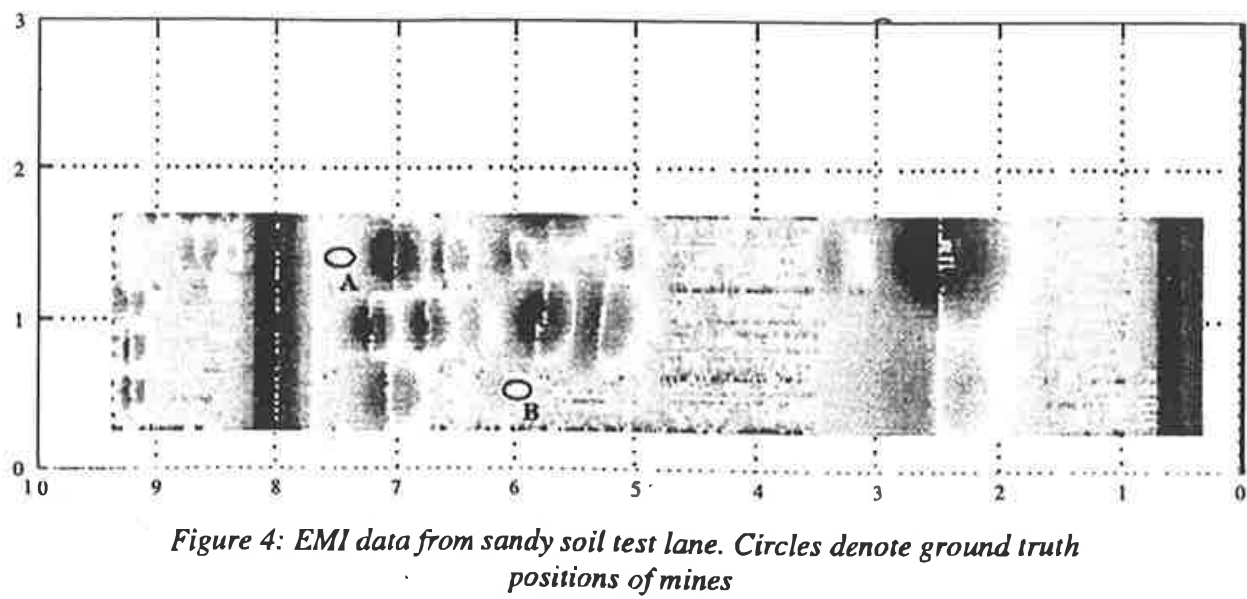


Figure 4 shows a typical data set that was collected using the continuous wave, hand carried metal detector. The anti-symmetric patterns that occur near target objects are caused by the detector receiver coil geometry. Both handheld systems that are used, produce a signal that crosses zero when moved over an object. This property is used as the basis for the detection algorithm.

The sensor data that are shown in Figure 2 through 4 illustrate the advantages that can be obtained through sensor fusion. The non-metal mine that is buried just below the surface at position A is clearly visible in the infrared image, but it can be barely discerned in GPR en EMI data. The non-metal mine that is buried $10 \mathrm{~cm}$ below the surface at position B is clearly visible in the GPR data, but it can be barely discerned in the TIR en EMI data.

\subsection{Sensor fusion parameter settings}

The choice of suitable parameter settings is one of the main issues in the optimisation of the sensor fusion architecture that is under development in the HOM-2000 project. Essential fusion parameters occur in confidence level discretisation and voting logic fusion.

In order to reduce the complexity of the parameter optimisation process, only logic tables based on binary input and output arguments are evaluated. As a consequence, the confidence level discretisation process is reduced to a thresholding mechanism. For each sensor, the threshold level is selected from a set of ten different values. These values are chosen such that corresponding points on the receiver operator curve of an individual sensor are approximately uniformly distributed between maximum and minimum detection probability. This means that for each voting logic table approximately 1000 parameter combinations are evaluated.

\subsection{Results}

The evaluation of sensor fusion results relies on automatic comparison of sensor fusion output and ground truth object position information. In the evaluation of the performance of the mine detection system we have to take into account both the number of correctly detected mines and the number of false alarms. According to our definition a mine is correctly detected if an alarm is found within an ellipsoid area of predetermined size around the centre of a mine. The size of the ellipsoid area depends on the size of the object and the accuracy of sensor position measurements.

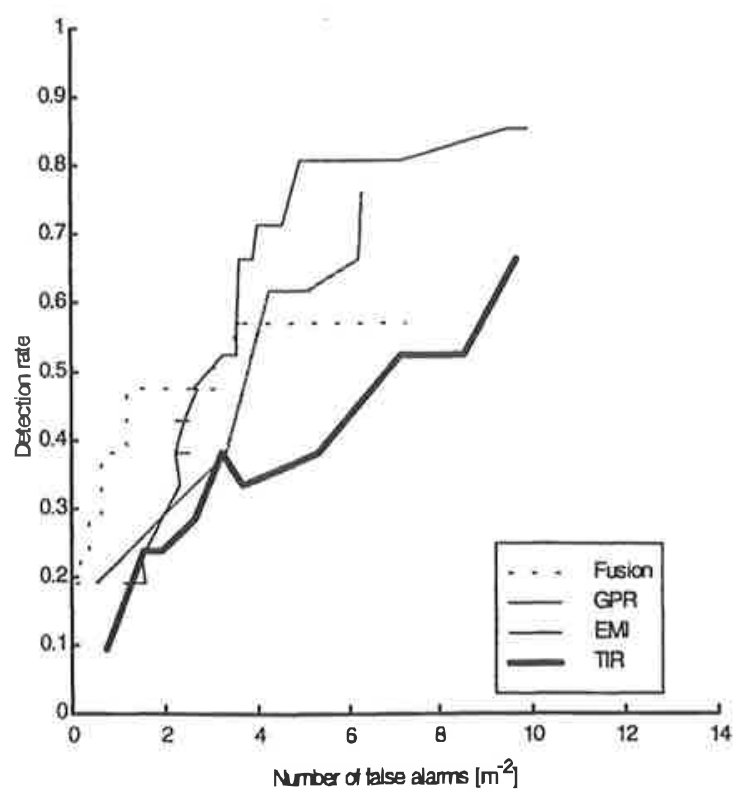

Figure 5: ROC of GPR, EMI, TIR and fusion performance in sand lest lane. Fusion logic based on two out of three sensors.

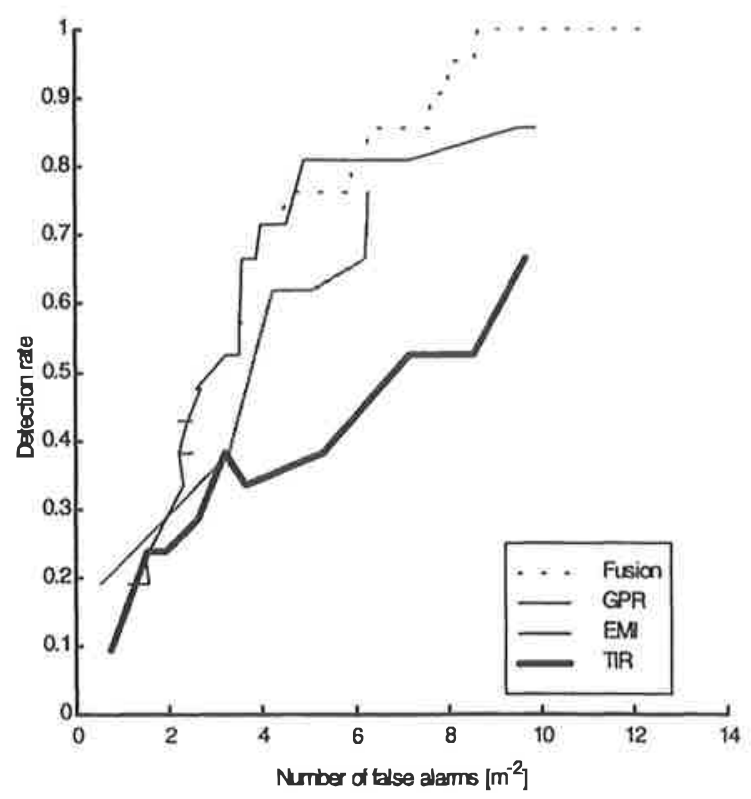

Figure 6: ROC of GPR, EMI, TIR and fusion performance in sand test lane. Fusion logic based on one out of three sensors. 
The computation of false alărms is based on the following procedure ${ }^{8}$ : In each detection region the number of mines is calculated. For each mine the effective false alarm region size is reduced by the size of the standard dig area. In our experiment the standard dig area measures $25 \times 25 \mathrm{~cm}$. If the effective size of a false alarm region is still above the standard dig area, each dig area that fits in the detection region is counted as one false alarm. By using this false alarm counting technique we can avoid misleading performance interpretations that would occur if large alarm regions were counted as single false alarms.

An analysis of the mine detection performance that can be achieved on the sandy soil test lane is shown in Figure 5 and 6. The analysis of the detector performance is based on the specific part of the test lane area that is covered by all of the three sensors. In this part of the test lane 21 mines are buried. The dashed ROC (Receiver Operator Characteristic) curve in Figure 5 is based on a fusion logic table that generates positive detection output signals only in those situations were at least two individual sensors provide supporting evidence. In the sandy soil test lane experiment, this type of fusion results in a reduction of the false alarm rate as compared to the individual sensors. The dashed ROC curve in Figure 6 is based on a fusion logic table that generates positive detection output signals if at least one individual sensor provides supporting evidence. In the sandy soil test lane experiment, this type of fusion results in an improved detection rate as compared to the individual sensors.

\section{CONCLUSIONS}

During HOM-2000 phase A, we have conducted a literature study on several different types of sensor fusion techniques. Based on theoretical arguments we have selected weighted voting as the most suitable core fusion algorithm. In addition to the core fusion algorithm, feature extraction algorithms were developed. Based on the analysis of sensor data gathered at the test facility, several situations have been identified that illustrate the benefits of sensor fusion.

\section{ACKNOWLEDGEMENTS}

The authors are grateful for the fruitful discussions and collaboration with H. Lensen, A. Schoolderman, A. Wilbers, W. de Jong, G. Beckers, J. Sluiter, Y. Janssen and all other participants in phase A of the HOM-2000 project. The HOM-2000 project phase A has been performed by TNO-FEL under contract of the Dutch ministry of Defense.

\section{REFERENCES}

1. E. Waltz and J. Llinas, Multisensor datafusion, Artech House, Norwood, 1990.

2. W. Altshuler, A.M. Andrews and A. Sparrow, "Mine and UXO Detection: Measures of Performance and their implication in Real-World Scenarios", Proc. SPIE Vol. 3079, Detection and Remediation Technologies for Mines and Minelike Targets II, p. 281-292, 1997.

3. A.M. Andrews, V. George and W. Altshuler, "Quantifying performance of mine detectors with fewer than 10,000 targets", Proc. SPIE Vol. 3079, Detection and Remediation Technologies for Mines and Minelike Targets II, p. 273-280, 1997.

4. B.A. Baertlein and A. Gunatilaka, "Improving detection of buried land mines through sensor fusion", Proc. SPIE Vol. 3392, Detection and Remediation Technologies for Mines and Minelike Targets III, p. 1122-1133,1998.

5. D.L. Hall, Mathematical Techniques in Multisensor Datafusion, Artech House, 1992.

6. A.L. Klein, Sensor and data fusion concepts and applications, SPIE Optical Engineering Press, Bellingham 1993.

7. A.L. Klein, "A Boolean Algebra Approach to Multiple Sensor Voting Fusion”, IEEE Transactions on Aerospace and Electronic Systems, vol. 29, pp.317-326, April 1993.

8. E. den Breejen, K. Schutte and F. Cremer, "Sensor fusion for anti personnel landmine detection, a case study", to be published in proceedings of AeroSense99.

9. W. de Jong, H.A. Lensen and Y.H.L. Janssen, "Sophisticated test facilities for land mine detection", to be published in proceedings of AeroSense99.

10. J. Rhebergen and P. Zwamborn, "Mine-detection test facilities at TNO-Fel test location Waalsdorp", NATO RTO lectures series 214, Advanced Pattern Recognition Techniques, Canada, 1998. 